\title{
Indicação de biomateriais em alvéolos pós extração previamente à instalação de implantes
}

\author{
Indication of biomaterials in post-extraction socket prior to implant placement \\ Karin Gisel Apaza Bedoya', Camilo Andrés Villabona López ${ }^{1,2}$, Gabriela Peñarrieta Juanito', Renato Yassutaka Faria Yaedu \\ Cesar Augusto Magalhaes Benfatti ${ }^{1}$
}

Para citar este artículo: Apaza-Bedoya K, Villabona CA, Peñarrieta JG, Yaedu RYF, Benfatti CAM. Indicação de biomateriais em alvéolos pós extração previamente à instalação de implantes. UstaSalud, 2017;16: 52-68

Licencia Creative Commons

\section{(c) (1) $(9)$} lo tanto, los lectores pueden acceder libremente a los artículos en su formato .pdf, igualmente podrán descargarlos y difundirlos; sin embargo no podrán modificarlos o alterarlos, adicionalmente se debe reconocer la autoría de las personas que figuran en las publicaciones, pero estas no podrán comercializadas.

\begin{abstract}
${ }^{1}$ Master program in Implantology Federal University of Santa Cataria, Florianópolis, Brazil. Centro de ensino e pesquisa em implantes dentarios (CEPID), Universidade Federal de Santa Catarina, Florianopolis, Brasil

2 Universidade Santo Tomás, Bucaramanga, Santander, Colômbia

${ }^{3}$ Faculdade de Odontologia de Bauru, Universidade de São Paulo, Brasil
\end{abstract}

Correspondence to:

Karin Gisel Apaza Bedoya

E-mail: karin_ab@hotmail.com

\section{RESUMO}

O presente trabalho descreve os processos fisiológicos envolvidos no processo de cicatrização alveolar, assim como as mudanças dimensionais que suscitam-se depois da perda do elemento dentário. Desta maneira, mostra-se a importância da utilização de biomateriais. Os quais favoreçam a posterior reabilitação com implantes osseointegrados. A presente revisão de literatura, a partir de critérios de inclusão e exclusão, propõe o estudo dos biomateriais encontrados na literatura e busca a avaliação criteriosa das suas propriedades, vantagens e limitações na indicação de alvéolos pós-extração.

Palavras-chave: Extração dentária, enxerto ósseo, biomaterial.

\section{ABSTRACT}

This study describes the physiological processes involved in the healing process, as well as the dimensional changes that occur after tooth loss. So the importance of the use a biomaterial as a socket filler is shown, which will improve future results for implant rehabilitation. The present literature review, based on inclusion and exclusion criteria, aims to evaluate biomaterial's properties, advantages and limitations for post-extraction socket.

Keywords: Tooth extraction, bone transplantation, biomaterial. 


\section{INTRODUÇÃO}

Sabe-se que a perda do dente leva à reabsorção fisiológica do rebordo alveolar e que a maior parte das mudanças dimensionais ocorrem nos primeiros meses depois da extração dentária. Desta forma, as pesquisas científicas são direcionadas a encontrar um biomaterial ideal que sirva como um substituto ósseo, promovendo a manutenção do volume do rebordo alveolar após a extração. Além disso, o biomaterial deve ser capaz de permanecer o tempo suficiente para permitir a nova formação do osso, visando a instalação de implantes e favorecendo a posterior reabilitação implantosuportada, atingindo assim, resultados estéticos e funcionáis favoravéis. ${ }^{1,2}$

A diversidade de biomateriais encontrados na literatura dificulta a escolha do material mais adequado para cada caso clínico. Por esta razão, é importante conhecer o biomateria que será utilizado, para atingir o objetivo desejado no menor tempo possível., ${ }^{2,3}$

Neste contexto, o presente estudo visa realizar uma revisão da literatura com relação à utilização dos principais biomateriais, descrevendo suas propriedades, vantagens e limitações mais utilizados em alvéolos pós-extração, para a posterior reabilitação com implantes osseointegrados.

\section{MATERIAIS E MÉTODOS}

Realizou-se uma busca na base de dados de PubMed (NCBI) das palavras-chave: "Tooth socket" , "Alveolar process", "Tooth extraction", "Alveolar ridge preservation", "Bone graft", Biomaterials, "Bone substitutes”; Allograft; Xenograft; Alloplastic.

Os critérios de inclusão foram artigos em idioma inglês publicados desde janeiro de 2004 até outubro 2013, estudos feitos em alvéolos pós extração em humanos ou animais. E estudos que avaliassem histologicamente o comportamento do biomaterial nos alvéolos pós extração para posterior instalação de implantes.

Os criterios de exclusão foram artigos em outros idiomas que não sejam inglês, estudos in vitro ou estudo de relato de caso clínico. Foram excluídos os estu- dos realizados em cavidades ou defeitos criados, assim como também estudos que incluíssem a instalação de implantes imediatos.

\section{Revisão de literatura}

\section{Tecido ósseo}

O osso é um tecido conjuntivo especializado vascularizado e dinâmico que se modifica ao longo da vida do organismo. A maioria dos ossos da face, é formada por ossificação intramembranosa. A remodelação óssea ocorre a partir da reabsorção local e do processo aposicional de formação óssea. ${ }^{1,2}$

O tecido ósseo, estruturalmente é constituído por uma matriz orgânica (30\%) e uma inorgânica (70\%). A matriz orgânica contém principalmente o colágeno tipo I que constitui $90 \%$ da proteína óssea total, e a porcentagem restante corresponde à proteínas não estruturais como os fatores de crescimento (BMPs, TGF- $\beta$, IGF), proteínas sanguíneas, glicoproteínas, proteoglicanas (PGs) e as sialoproteinas ósseas. A porção inorgânica do osso é constituída de fosfato de cálcio principalmente de cristais de Hidroxiapatita, embora o cálcio possa ser substituído por outros cátions bivalentes. ${ }^{2,3}$

O osso maduro pode se apresentar como um osso compacto na camada mais externa e dura do osso, denominado osso cortical. O osso cortical tem uma estrutura morfológica única contendo os osteons (sistemas Haversianos), onde lamelas de matriz óssea se dispõem de forma concêntrica em torno de um canal central (canal de Havers). Dentro das lamelas observam-se espaços pequenos, as lacunas, tendo osteócitos em seu interior. Os canais de Havers se comunicam entre si através de canais dispostos perpendicularmente (canal de Volkman). ${ }^{2}$

A parte interna do osso, é constituída pelo osso esponjoso que contém medula óssea ou tecido conjuntivo embrionário. O tecido ósseo esponjoso é responsável en torno de $70 \%$ do metabolismo ósseo. A comunicação celular direta ou indireta entre os diferentes constituintes celulares (osteoblastos, osteócitos e pré-osteoclastos) é essencial para a remodelação óssea a qual é regulada pelo balanço entre dois fenótipos opostos: osteoclastos e osteoblastos. Sendo que o 
fenótipo osteogênico secreta a matriz óssea no sitio de reabsorção do fenótipo osteoclástico. ${ }^{2}$

\section{Preservação do osso alveolar}

A preservação do rebordo alveolar surgiu entre os anos 70 e 80, com a ideia de maximizar a retenção das próteses removíveis e, desta forma, melhorar o grau de satisfação dos pacientes. A preservação alveolar abrange os procedimentos que tem como objetivo limitar o efeito negativo da reabsorção pós-exodontia, visando manter o contorno dos tecidos duros e moles. A preservação alveolar promove a formação óssea no alvéolo e, desta maneira, facilita a instalação do implante numa posição protéticamente guiada.

Primeiramente, deve-se identificar e documentar os fatores da história médica do paciente que comprometeram a cicatrização tais como fumo, diabetes não controlado adequadamente, insuficiência hepática, uso abusivo de drogas e álcool, usuários por longa data de corticosteroides e pacientes de extrema idade. ${ }^{4}$

Outro aspecto importante é conhecer a história do dente, saber a patologia que leva o dente à extração. ${ }^{4}$ Tramentos ortodônticos, como a vestibularização dos dentes, podem mudar a espessura do tecido mole e do osso subjacente. Da mesma maneira, uma expansão rápida do arco pode levar a uma redução da tábua vestibular e aumentar a possibilidade de uma deiscência. ${ }^{5}$ Uma avaliação periodontal é necessária, assim como o controle das infecções periodontais na área com o fim de avaliar com precisão os contornos gengivais e projetar o resultado final desejado do tratamento. Uma recessão gengival indicaria uma deiscência no osso subjacente, o que sugere a possibilidade de outros tratamentos como tração coronária antes de uma extração dentária. A mobilidade dentária indicaria também uma deiscência. ${ }^{5}$

A preservação alveolar comença desde uma extração minimamente invasivo, razão pela qual deve-se avaliar a necessidade da utilização de retalho vestibular. A tábua óssea vestibular é frequentemente fina e ao abrir um retalho vestibular o suprimento sanguíneo do periósteo fica alterado. Em caso de necessidade do uso de membrana, ela se adapta nas paredes do alvéolo. Em situações onde se tem uma perda vertical de mais de $5 \mathrm{~mm}$ do osso vestibular é indicado elevar retalho vestibular para adaptação da membrana e uma correta degranulação na vestibular do alvéolo. ${ }^{6} \mathrm{~A}$ elevação do retalho diminuiria a habilidade das células do periósteo de regenerar osso, enquanto que um periósteo intacto mantém o potencial osteogênico. ${ }^{7}$

Fatores estéticos também como linha do sorriso, linha da margem gengival dos dentes adjacentes, assimetrias e comprimentos de papilas devem ser considerados, permitindo assim um planejamento abrangente e prevendo os possíveis resultados estéticos que serão alcançados segundo o caso clínico. ${ }^{4}$

Pietrokovski e Massler ${ }^{8}$ (1969) estudaram a quantidade de perda do tecido depois da exodontia de um dente unilateral usando modelos de gesso e concluíram o osso da vestibular tanto da maxila como da mandíbula foi reabsorvido consideravelmente mais que o osso palatino/ lingual. Araujo et al. ${ }^{9}$ (2005) confirmam isto em um estudo com cães ao qual demostraram que as mudanças no osso alveolar eram assimétricas sendo maior no osso vestibular que no palatino/lingual. Sendo que, o osso vestibular é mais fino e que, como em todo o alvéolo, esta constituído por uma estrutura óssea chamada de osso fascicular, a qual é dento-dependente, assim com a extração dentaria é evidente a consequente perda desta estrutura, chegando até $2 \mathrm{~mm}$ de perda vertical.

Estudos inferem uma maior perda óssea nos primeiros 3 a 4 meses depois da exodontia. ${ }^{9-11}$ Ao exame radiográfico, Schropp et al. ${ }^{11}$ (2003) confirmou que a formação do osso dentro do alvéolo ocorre simultaneamente com a perda da altura do rebordo. Em outro estudo foi constatado que aproximadamente de 5 a 7 $\mathrm{mm}$ de osso horizontal ou bucolingual é reabsorvido e isto ocorre tão somente dentro do $6^{\circ}$ a $12^{\circ}$ mês. A reabsorção apicocoronal ou vertical reportada é de $2.0 \mathrm{a}$ $4.5 \mathrm{~mm} .{ }^{12}$ A maioria das mudanças dimensionais ocorrem durante os primeiros 3 a 4 meses, sendo maiores em extrações multiplas do que em extrações unitárias. Porém, uma reabsorção lenta, porém contínua, ocorre pelo resto da vida do paciente..$^{11,12}$

A literatura mostra que a mudança dimensional em região do molar é maior que na região anterior ${ }^{8,13}$, contudo, na região anterior, esta redução é crítica visto que compromete a estética rosa e branca da futura reabilitação protética. Estudos mostram que 
na região anterior encontra-se uma parede vestibular fina, menor que $1 \mathrm{~mm} .{ }^{63,64} \mathrm{Na}$ zona estética maxilar anterior, observou-se uma perda volumétrica severa nos primeiros 6 meses depois da extração e continua ao longo do tempo com um adicional de $11 \%$ nos 5 anos seguintes. ${ }^{13}$

O estudo de Rasperini et al. (2010) ${ }^{14}$ mostra uma tendência de que procedimentos de levantamento de seio são mais requeridos quando os alvéolos não são enxertados devido a perda óssea vertical.

Nevins et al. (2006) ${ }^{15}$ concluiram que é prudente colocar uma substância osteocondutora nos alvéolos de dente com raizes prominentes para evitar a perda do osso vestibular comprometendo o tratamento com implantes.

Block e Jackson (2012) ${ }^{16}$ acreditam que na presença de alvéolos com defeitos na tábua vestibular, deve-se avaliar a extensão, sendo que de 3 a $6 \mathrm{~mm}$ pode-se utilizar osso mineralizado e após 16 semanas instalar o implante, ou optar por instalação do implante simultânea ao enxerto protegido com membrana. Nos defeitos maiores do que $6 \mathrm{~mm}$ o local deve ser enxertado, para depois da consolidação do enxerto ser instalado o implante.

A cicatrização fisiológica requer a presença de ligamento periodontal devido a presença de células proliferativas que migram no centro do alvéolo e se diferenciam em osteoblastos promovendo a neoformação óssea. ${ }^{17}$ Alguns estudos apoiam esta hipótese pela retenção do coágulo nos primeiros estágios da cicatrização. Entretanto outros estudos mostram que o alvéolo deve ser debridado para remover tudo que possa interferir na cicatrização e até deve-se realizar perfurações nas paredes do alvéolo facilitando a vascularização no alvéolo. ${ }^{18}$

\section{Cicatrização nos alveolos pós extração}

Os alvéolos após extração cicatrizam por segunda intenção, o que significa que um espaço é mantido entre as margens de uma incisão, laceração ou neste caso, de fragmentos ósseos. ${ }^{19}$

Eventos biológicos ocorrem durante a cicatrização dos alvéolos pós extração e cinco estágios da cica- trização são descritos: O primeiro estágio é quando o alvéolo é preenchido pelo coágulo de sangue, que consiste em eritrócitos e leucócitos em uma rede de fibrina. No segundo estágio o tecido de granulação, rico em novas estruturas vasculares neoformadas e células inflamatórias como leucócitos e macrófagos, substitui o coágulo ao redor do $4^{\circ}$ ao $5^{\circ}$ dia. No terceiro estágio o tecido de granulação gradativamente é substituído pelo tecido conjuntivo, onde se forma a matriz provisória $\left(14^{\circ}-16^{\circ} \mathrm{dia}\right)$, a qual apresenta células mesenquimais densas, fibras de colágeno e vasos ou apenas poucas células inflamatórias espalhadas. No quarto estágio forma-se o tecido ósseo, aparecem calcificações da matriz osteóide, na base e na periferia do alvéolo no $7^{\circ}$ e $10^{\circ}$ dia. No quinto estágio observa-se osso lamelar e medular, ou seja, lamelas de osso maduro, osso mineralizado abrigando osteócitos por espaços medulares ricos em vasos, adipócitos, células mesenquimais e células inflamatórias. ${ }^{12,19,20}$

O fechamento epitelial do alvéolo completa-se no $24^{\circ}$ ao $35^{\circ}$ dia. Preenchimento ósseo considerável ocorre entre a $5^{\mathrm{a}}$ e $10^{\mathrm{a}}$ semana. Apenas na $16^{\mathrm{a}}$ semana, ocorre o preenchimento completo do alvéolo por osso neoformado e existe uma pequena evidência de atividade osteogênica. ${ }^{12}$

O estudo de Cardaropoli, Araujo e Lindhe (2003) ${ }^{21}$ mostra a cicatrização dos alvéolos após exodontia da raiz distal de pré-molares em cães. Observou-se que o tecido ósseo preenche o alvéolo em um mês, que a crista cortical inclui tecido ósseo e osso lamelar formado em 3 meses e após o tecido ósseo foi gradativamente substituído por osso lamelar e medular.

\section{Biomateriais}

Segundo Carvalho $(2011)^{22}$, os biomateriais devem possuir as propriedades descritas a seguir: Não induzir a formação de trombos como resultados do contato entre o sangue e o biomaterial, não induzir resposta imune adversa, ser atóxico, não ser carcinogênico, não atrapalhar a angiogênese e não produzir resposta inflamatória aguda ou crônica.

As características da superfície dos substitutos ósseos são determinadas pela composição química, microporosidade, rugosidade de superfície, cristalização e tamanho do cristal. ${ }^{3}$ 
Uma característica importante é a porosidade do arcabouço utilizado. Sabe-se que o osso cortical apresenta poros entre $1 \mu \mathrm{m}$ e $100 \mu \mathrm{m}$, enquanto o trabecular entre $200 \mu \mathrm{m}$ a $400 \mu \mathrm{m}$. A dimensão, fração de volume e interconectividade dos poros afetam criticamente a difusão de nutrientes e dejetos metabólicos, adesão e migração celular, expressão de proteínas e formação tecidual necessárias ao reparo e regeneração óssea. Por isso é ideal em um biomaterial a presença de poros interconectados com dimensões de $100 \mu \mathrm{m}$ a $500 \mu \mathrm{m}$, poros menores usualmente resultam na formação de tecido fibrovascular. Outra característica importante é a taxa de reabsorção ou degradação dos compostos já que é necessária uma boa relação reabsorção/ deposição óssea. Os arcabouços com degradação acelerada podem restituir uma cavidade tecidual, enquanto matérias que não são reabsorvidos adequadamente impedem uma deposição natural e limitam a osseointegração do enxerto. O biomaterial compatível deve evitar as complicações da necessidade de imunossupressão. Também deve ser biocompativel e promover condutividade para a adesão e proliferação de células comprometidas. A neovascularização pelo substituto tecidual é importante para o fornecimento de oxigênio e transporte biomolecular ao sitio implantado. Da mesma maneira, o biomaterial ideal deve ser capaz de suportar forças mecânicas no sitio da implantação. Outra necessidade refere-se à taxa de degradação, formando metabólitos não tóxicos, facilmente metabolizados ou excretados. ${ }^{2}$

Como foi descrito por Carvalho ${ }^{22,23}$ os biomateriais em implantodontia se classificam em:

Quanto à origem: Homógenos / Alogénos: da mesma espécie, DFDBA, FDBA (osso congelado, seco e/ ou desmineralizado, conhecido como osso liofilizado). Autógeno: obtido do mesmo organismo, osso cortical, medular, plasma rico em plaquetas (PRP), plasma rico em fibrina (PRF). Xenógeno / Heterólogo: de diferentes espécies, coral, algas. Considerados semissintéticos por terem a obrigatoriedade de passar pelo processo industrial de purificação. Aloplásticos: sintéticos, vidro bioativo, carbonato de cálcio, sulfato de cálcio, fosfato de cálcio (calciometafosfato, monocalciofosfato, calciohidrogenofosfato, calciopirofosfato, octacalciofosfato, tricalciofosfato (TCP), pentacalciohidroxifosfato ou hidroxiapatita, tetracalciofosfato).
Quanto à resposta biológica: Biotolerado: apresentam tecido conjuntivo fibroso na interface. Bioinerte: caracterizado por neoformação óssea de contato. Bioativo: induz uma reação físico - química.

Quanto à característica física: Anorgânico ou inorgânico ou mineralizado: os componentes são removidos e a matriz inorgânica é preparada na forma de grânulos. Desmineralizado: os componentes inorgânicos e celulares são removidos, permanecendo os componentes da matriz extracelular. Fresco: biomaterial obtido sem nenhum tipo de processamento.

Quanto à propriedade biológica: Osteoconductor: conduz o desenvolvimento de novo tecido ósseo através de sua matriz de suporte (arcabouço). Osteoindutor: a osteogênese é induzida e envolve a formação de novo osso a partir da diferenciação de células em osteoprogenitoras. Osteogénico: as células ósseas vivas e remanescentes no enxerto mantém a capacidade de formar matriz óssea. Osteopromotor: são meios físicos (membranas e barreiras) que promovem o isolamento anatômico de um local, permitindo a seleção e proliferação de um grupo de células.

A seleção do biomaterial é uma tarefa complexa, a multiplicidade de materiais disponíveis dificulta a escolha, assim para atingir uma solução satisfatória deve-se considerar ${ }^{2}$ : Aplicação: é preciso ter clara qual a aplicação que se pretende; Requisitos importantes: o que é exigido do biomaterial para a aplicação definida; priorização: quais requisitos são fundamentais e quais são desejáveis; escolha técnica: possíveis biomateriais a utilizar e escolha final: dentro das soluções técnicas se faz a escolha a partir do aspecto econômico e/ ou aspectos ambientais.

\subsection{Alogénos}

O enxerto alográfico é armazenado usualmente nos "bancos de ossos" e exige-se um controle periódico do material. Podem ser utilizados como osso fresco congelado (fresh frozen bone, FFB), osso alográfico liofilizado (freeze- dried bone allograft, FDBA) ou osso alográfico liofilizado desmineralizado (demineralized freeze-dried bone allograft, DFDBA). FFB não é comumente utilizado devido a sua reação imunológica. ${ }^{3}$ 
A escolha deste biomaterial deve ser baseada na facilidade de entrega, custo, consistência na aparência, e a qualidade do banco de ossos. Este tipo de biomaterial tem como desvantagem as possíveis opiniões religiosas ou culturais que podem impedir a enxertia nos pacientes e a possível transmissão de doenças. ${ }^{16}$

O FDBA e DFDBA mostram biocompatibilidade e contém moléculas osteoinductivas como as proteínas morfogenéticas ósseas (BMPs). Durante o processo de desmineralização FDBA perde parte de sua estabilidade mecânica, e DFDBA deve combinar-se com algum material que mantenha o espaço caso seja colocado em uma estrutura não contida pela mesma. DFDBA pode apresentar-se com diferentes concentrações de BMPs e a osteocondutividade varia de acordo com a mesma. $^{16}$

O DFDBA pode ser processado sendo imerso em Etanol $100 \%$ para a remoção de gorduras, congelado com nitrogênio, liofilizado e granulado em partículas de diferentes tamanhos. A liofilização por tempo prolongado diminui a antigenicidade. No processo de desmineralização utiliza-se $0.6 \mathrm{~N}$ de ácido clorídrico $(\mathrm{HCl})$ ou ácido nítrico, o que assegura o estado livre de doenças. $\mathrm{O} \mathrm{HCl}$ remove os sais de cálcio e fosfato, retendo o colágeno e teoricamente expondo à BMP. Depois da lavagem e desidratação, ele é irradiado ou esterilizado à frio. ${ }^{16}$

Uma forma de processamento do FDBA é usando o método Tutoplas, que oferece como resultado um osso mineralizado com a matriz de colágeno intacta. Este processo envolve várias lavagens para remover gorduras, material celular e proteínas não colágenas, eliminando proteínas que possam ser patogênicas e preservando a atividade indutiva proteica e o padrão trabecular natural. Depois de ser desengordurado com acetona, passa por um tratamento osmótico, tratamento oxidativo, é desidratado para ser finalmente irradiado por raios gama. ${ }^{16}$

No estudo de Becker, Becker e Caffese $(1994)^{24}$ observou-se que os alvéolos enxertados com partículas de 250 - $500 \mu \mathrm{m}$ de DFDBA, num período de tempo de 3 a 13 meses, apresentaram partículas do biomaterial mortas, rodeadas de tecido conjuntivo não inflamatório e sem evidência de atividade osteoclástica ou osteoblástica. No momento do trefinamento apresen- tou-se um osso de fácil remoção, contrastando com os outros alvéolos estudados enxertados com osso autógeno os quais apresentaram-se com sinais de formação óssea ativa.

Brownfield e Weltman ${ }^{25}$ estudaram alvéolos enxertados com uma matriz de osso desmineralizado osteoindutor com lascas de osso esponjoso (Dynablast) e um grupo controle sem tratamento, ambos cobertos com membrana de colágeno reabsorvível. Depois de 10 a 12 semanas a tomografia computadorizada cone beam (CBCT) mostrou uma redução média vertical de $0.8 \mathrm{~mm}$ para o grupo controle e $1.2 \mathrm{~mm}$ para o grupo teste, enquanto a redução na espessura $1.6 \mathrm{~mm}$ para o grupo testado e $2.1 \mathrm{~mm}$ para o sitio controle. Este estudo encontrou histologicamente uma neoformação óssea em $37.4 \%$ e com a micro tomografia computarizada (microCT) $44.9 \%$ no grupo teste, e histologicamente $35.5 \%$ e com microCT $39 \%$ no grupo controle. Biomaterial residual foi encontrado em $4.5 \%$ nos cortes histológicos e $2.4 \%$ com microCT.

De Scheyer, Schupbach e McGuire ${ }^{26}$ estudaram a formação óssea examinando histologicamente alvéolos preenchidos com DynaBlast (Keystone dental), osso esponjoso mineralizado em três períodos distintos. Observou-se que em 6 semanas já havia atividade osteoblástica e podia observar a regeneração precoce de tecido ósseo coronal ao osso apical denso nativo, em 12 semanas observou-se novo tecido ósseo formado ao redor das partículas demineralizadas do aloenxerto, regeneração óssea no terço médio e apical e em 24 semanas observou-se regeneração óssea em quase todo o espécime.

No estudo de Beck e Mealey ${ }^{27}$, observou-se alvéolos enxertados com partículas de $250-1000 \mu \mathrm{m}$ de osso alogéno mineralizado e osso liofilizado esponjoso, em dois tempos, depois de 14 semanas e 27 semanas. No grupo das 14 semanas encontrou $41.8 \%$ de osso vital e $15.3 \%$ de biomaterial residual. Neste grupo observou alguns pacientes com defeito ósseo numa parede onde foi colocado além do enxerto uma membrana, mostrando $19.3 \%$ de osso vital neoformado e $26.8 \%$ de biomaterial residual. Enquanto à reabsorção horizontal, no grupo de 14 semanas observou $1.47 \mathrm{~mm}$ (17.3\%), e no grupo de 27 semanas $1.43 \mathrm{~mm}$ (15.3\%). $\mathrm{Na}$ reabsorção horizontal menos de $1 \mathrm{~mm}$ foi observado em ambos os grupos, ressaltando um ligeiro ganho 
de altura na vestibular dos alvéolos do grupo de 27 semanas $(0.32 \%)$ não estatisticamente significativo.

No estudo de Lasella et al. ${ }^{28}$ dois grupos foram estudados, um grupo controle com alvéolo sem nenhum tratamento, e outro grupo enxertado com osso alógeno liofilizado hidratado com tetraciclina (FDBA) mais membrana colágena. Observou uma reabsorção alveolar horizontal menor no grupo FDBA (1.2 \pm 0.9 $\mathrm{mm}$ ) que no grupo controle $(2.6 \pm 2.3 \mathrm{~mm})$; e um ganho de osso em sentido vertical no grupo FDBA ( $1.3 \pm$ 2.0 ) versus a perda óssea horizontal no grupo controle $(0.9 \pm 1.6 \mathrm{~mm})$ dando uma diferença estatisticamente significativa de $2.2 \mathrm{~mm}$. Os estudos histológicos revelaram uma maior presença de osso no grupo FDBA que no grupo controle, o grupo controle apresentou maior quantidade de espaços trabeculares. Cortes histológicos do grupo FDBA incluíram osso vital e não vital e fragmentos de FDBA.

Wood e Mealey ${ }^{29}$ compararam os alvéolos enxertados com FDBA e DFDBA. Depois de 19 semanas, em ambos grupos encontraram uma perda vertical do rebordo de $1 \mathrm{~mm}$ e horizontal de aproximadamente 2 $\mathrm{mm}$. Nos cortes histológicos observaram $81.26 \%$ de osso neoformado vital e $18.74 \%$ de biomaterial residual com DFDBA, e $50.63 \%$ e $49.37 \%$ correspondentemente com FDBA.

A literatura mostra que o aloenxerto associado a uma membrana não absorvível precisa de um fechamento gengival, em caso de exposição deve-se remover 6 semanas depois a colocação. ${ }^{16}$

Lee et al. ${ }^{30}$ compararam alvéolos enxertados com osso bovino alógeno mineral Bio-Oss (DBBM), aloenxerto esponjoso irradiado (ICA), aloenxerto solvente desidratado (SDA). O DBBM mostrou maior efeito osteocondutivo que ICA e SDA, e também um contato mais íntimo entre o novo osso formado e o biomaterial que os demais grupos, produzindo uma estrutura óssea mais rígida. Nos cortes histológicos com o ICA, depois de 4 a 6 meses, mostraram a presença de células inflamatórias nas áreas onde as partículas são reabsorvidas por osteoclastos. Nos cortes histológicos com SDA obteve-se um osso trabecular delgado, e o novo osso formado e irregular e separados entre si.

\subsection{Xenógenos}

Conhecidos também como xenoenxertos, são materiais retirados de outras espécies animais empregados clinicamente em humanos como cerâmicas naturais (descartando a porção biológica e mantendo apenas a estrutura óssea mineralizada) ou de forma semelhante as matrizes ósseas desmineralizada. Eles representam $50 \%$ do mercado mundial de enxertos ósseos. ${ }^{2}$

Matrizes ósseas desmineralizadas podem ser produzidas através da descalcificação de osso cortical e são normalmente obtidas a partir de cadáveres. A fase mineral do osso pode ser facilmente extraída por imersão em $0.5 \mathrm{~N}$ a $0.6 \mathrm{~N}$ de ácido clorídrico (HCL). Este tratamento químico é um efetivo inativador viral, bactericida e extrator de elementos sanguíneos, oferecendo baixo risco de transmissão de doenças e imunogenicidade diminuída. $\mathrm{O}$ material extraído preserva convenientemente a estrutura trabecular e boa parte da porção orgânica da matriz extracelular, assim serve como um arcabouço biológico apesar da menor resistência estrutural decorrente da extração da porção mineral. A desmineralização óssea não elimina todos os fatores de crescimento contidos na porção biológica do osso processado. Assim, matrizes ósseas desmineralizadas são potencialmente ainda mais osteoindutivas do que os enxertos alógenos mineralizados. ${ }^{2}$

O colágeno contribui no auxilio da adesão e migração celular, deposição mineral, crescimento vascular e apresentação de fatores de crescimento proporcionando um ambiente favorável à regeneração óssea. Apesar das óbvias vantagens do colágeno, eles de modo geral podem apresentar imunogenicidade, o que limitaria seu uso clínico. As matrizes colágenas constituídas in vitro são menos densas, mais hidratadas e mecanicamente mais frágeis, proporcionando baixo suporte estrutural e de osteocondução. Por enquanto, os estudos clínicos conduzidos tem empregado o colágeno como um sistema de entrega ou matriz biológica auxiliar a outros biomateriais. ${ }^{2}$

Barone et al. ${ }^{31}$ se avaliaram o enxerto de osso porcino corticoesponjoso (CPB) em forma de partículas de porosidade alta e de diâmetro de 600 a $1000 \mu \mathrm{m}$. Neste estudo se comparou um grupo sem nenhum tipo de tratamento depois da extração dentaria (GC) e um grupo enxertado com $\mathrm{CPB}$ mais membrana colá- 
gena. Observaram sete meses depois, uma reabsorção horizontal menor no grupo CPB (4.3 $\mathrm{mm}$ vs $2.5 \mathrm{~mm})$ e uma reabsorção menor também na altura da tábua vestibular do grupo enxertado ( $3.6 \mathrm{~mm}$ vs $0.7 \mathrm{~mm})$. A biópsia dos sítios enxertados mostrou a presença de osso trabeculado, com uma alta mineralização e bem estruturado, podendo-se observar partículas do material enxertado. A biópsia dos sítios livres de enxerto mostrou também um osso bem estruturado com uma menor porcentagem de osso mineralizado. CPB é um osso porcino orgânico quimicamente tratado para remover os componentes orgânicos. Depois de esterilizado, pode-se utilizar este biomaterial sem causar resposta imune. A densidade dele proporciona estabilidade ao enxerto e larga durabilidade porque ele não reabsorve completamente. ${ }^{32}$

Hidroxiapatitas xenógenas, como aquelas derivadas de corais ou osso bovino, são usadas há mais de 20 anos no mercado. ${ }^{2}$

KIM et al. ${ }^{33}$ mostraram a diferença de reabsorção horizontal depois de 3 meses entre o grupo de alvéolos sem tratamento $(20.74 \%)$ e o grupo tratado com esponja colágena mais xenoenxerto Bio-Oss (14.26\%). Enquanto à reabsorção vertical, no grupo controle apresentou $6.83 \%$ e o grupo experimental apresentou $5.76 \%$.

Bio-Oss Collagen é uma mistura de grânulos de osso bovino desproteinizado (90\%) e fibras de colágeno tipo I (10\%), e tem sido usada amplamente no mundo.

No estudo de Heberer ${ }^{34}$ concluiu-se que a formação óssea avaliada histologicamente depois de 3 meses dos alvéolos preenchidos com Bio-Oss colágeno foi menor que nos alvéolos não enxertados. ( $25 \%$ vs $44 \%)$. Foi observado $15 \%$ de biomaterial residual e um osso com formação precoce nas áreas mais coronais que nas apicais (32\% vs $18 \%$ ) Os alvéolos ficaram expostos em ambos grupos e sem tratamento farmacológico, e não observou-se nenhum caso de inflamação ou infecção.

No estudo de Cardaropoli et al. ${ }^{35}$ observou-se que depois de 4 meses no grupo controle, sem nenhum tipo de tratamento pós extração, apresentou uma redução horizontal de $4.48 \mathrm{~mm}$ (33.48\%) e o grupo experimental, preenchido com Bio-Oss Collagen mais membrana de colágeno Bio-Gide exposta, apresen- tou uma redução de $1.04 \mathrm{~mm}$ (7.70\%). Observou-se também uma perda vertical para o grupo controle de $1.54 \mathrm{~mm}$ e para o grupo testado de $0.46 \mathrm{~mm}$. Nos cortes histológicos observou-se novo osso formado, no grupo controle $43.28 \%$ e para o grupo testado $26.34 \%$, o biomaterial residual (18.46\%) apresentou-se embebido em novo osso.

No estudo histológico de Lindhe et al. ${ }^{36}$ evidenciou-se que, depois de 6 meses, o grupo controle, recoberto com matriz colágena porcina (Mucrograft), apresentou maior porcentagem de osso lamelar e medular que no grupo testado, preenchido com Bio - Oss Collagen mais Mucograft, o que significaria que o processo de cicatrização de tecido duro é aparentemente demorado com a utilização deste biomaterial. O grupo com biomaterial mostrou no centro tecido conjuntivo rico em células, fibras e vasos; e nas laterais os muros com o osso neoformado mineralizado, o que sugere que o coágulo é formado entre a parede lateral do alvéolo e o biomaterial. Assim como em outros estudos $^{35,37}$ onde demostrou-se que a cicatrização dos alvéolos com bio-oss é mais rápida em áreas apical e lateral do alvéolo que nas áreas marginal e central.

Perelman - Karmon et al..$^{38}$ comparou o percentual de frações de área óssea nos alvéolos pós extração preenchida com osso mineral bovino com e sem membrana, concluindo que a utilização de membrana acrescentaria a quantidade de neoformação ósseas.

No estudo Crespi et al. ${ }^{39}$ estudaram grupos de alvéolos preenchidos com hidroxiapatita enriquecida em magnésio (MHA), osso porcino (PB) e sem nenhum tratamento $(\mathrm{C})$, mostrando maior porcentagem de osso vital no grupo MHA e PB que no C, não tendo diferença estaticamente significativa entre os grupos enxertados (MHA e PB).

Mardas, Chadha e Donos ${ }^{40}$ compararam um substituto sintético ósseo, Straumann Bone Ceramic (SBC) e um xenoenxerto bovino, Bio-Oss (DBBM), ambos protegidos com membrana de colágeno. Depois de 8 meses ambos grupos apresentaram osso neoformado no ápice e na parte coronal tecido conjuntivo fibroso denso ao redor dos biomateriais. Não tendo diferença estatisticamente significativa Ambos mostraram uma redução do rebordo no sentido bucolingual de 1.1 
mm (SBS) e de 2.1 (DBBM), a altura do rebordo foi mantida em ambos grupos.

Diferentes espécies de corais calcificados apresentam um esqueleto de carbonato de cálcio com a geometria similar ao osso esponjoso humano e microporos interconectados de 200 a $600 \mu \mathrm{m}$. O carbonato de cálcio de coral é transformado em HA por uma reação de troca hidrotermal com fósforo. Observou-se um potencial osteocondutivo menor que outros substitutos ósseos. ${ }^{3}$

Existe também outro grupo de xenoenxertos provenientes de algas marinhas que consistem num exoesqueleto calcificado feito de carbonato de cálcio. Ele é convertido em fluorhidroxiapatita através da reação de troca com fosfato de amônio a $700^{\circ} \mathrm{C}$, dando poros paralelos de $10 \mu \mathrm{m}$ e interconectados através de microperfurações, embora esta configuração não seja a ideal para a vascularização observou-se-se osteócitos aderidos no biomaterial. A hidroxiapatita proveniente das algas sofre uma reabsorção lenta por enzimas e degradação celular com menor taxa de degradação que os enxertos autógenos. ${ }^{3}$

\subsection{Aloplásticos}

Materiais sintéticos defendem a idéia de possíveis transmissões dos materiais semissinteticos, alegando o risco diante de doenças contagiosas. Por essa razão alguns clinicos, tem preferência pelos materiais obtidos laboratorialmente e sem contato com qualquer ser vivo. $^{23,41,42}$

Estes biomateriais tem ganhado a atenção de cientistas e clínicos nos últimos 40 anos, devido à possibilidade teórica de desenhar o material com características individuais para indicações clínicas específicas. ${ }^{3}$

O sulfato de cálcio $(\mathrm{CaP})$ é provavelmente o substituto ósseo mais antigo, foi descrito pela primeira vez em 1892. Atesta-se ainda a sua capacidade osteocondutiva, particularmente porque são materiais com boa resistência estrutural e rápida absorção (8-24 semanas). "Sulfato de cálcio" é o termo genérico que envolve muitos materiais. Isto se torna um problema para analisar estudos que não reportam a composição ou estrutura cristalográfica, não podendo dar uma conclusão objetiva do seu desempenho clínico. ${ }^{2}$

Cristalização é definida como a fração de $\mathrm{CaP}$ envolvida numa rede cristalina oposta a fração presente numa fase amorfa, e acredita-se que desempenha um importante papel na adsorção de proteínas, aderência celular e dissolução dos biomateriais. ${ }^{42} \mathrm{O}$ tamanho de cristal é normalmente dependente da temperatura a qual o CaP é sintetizado. A maior temperatura de sinterização gera maiores cristais. ${ }^{2}$

A forma, tamanho e a distância entre os cristais de apatita compõem a microporosidade do biomaterial (poros $\emptyset 10 \mathrm{~mm}$ ), e isso é importante para a penetração e a aderência de macromoléculas e fluido de tecidos. ${ }^{43}$

Os macroporos (poros $4100 \mathrm{~mm}$ ) proporcionam um arcabouço para a neoformação vascular e fixação de células formadoras de osso, com o pré-requisito de que esses poros estão interligados, como pode ser visto em osso nativo. $\mathrm{O}$ volume total dos micro e macroporos definem a porosidade. No CaP sintético, a porosidade geralmente não supera o $50 \%$ pelo comprometimento das propriedades mecânicas da cerâmica. ${ }^{2}$

Uma das vantagens dos cimentos de fosfato de cálcio é que ele pode ser produzida pelo próprio profissional durante a cirurgia. A reação de precipitação é geralmente exotérmica podendo alcançar temperaturas acima dos $37^{\circ} \mathrm{C}$. No entanto, bons resultados são observados para pequenos reparos em osso de menor carregamento. $^{2}$

Em geral, eles são preparados pela mistura de dois tipos de fosfatos de cálcio com uma solução aquosa. $\mathrm{O}$ produto desta mistura forma duas fases que serão precipitadas gradativamente, uma destas fases é a apatita, determinante da alta biocompatibilidade observada. $\mathrm{Na}$ medida em que os cristais crescem, o entrelaçamento de eles começa a induzir o endurecimento da pasta. $^{2}$

Ruga et al. ${ }^{44}$ encontraram ao estudar alvéolos preservados com sulfato de cálcio que depois de 4 meses $63.16 \%$ apresentava osso vital, $2.1 \%$ não vital, $4.74 \%$ tecido fibroso e $30 \%$ matéria amorfa, e nenhum resíduo de sulfato de cálcio. 
Clinicamente, o uso destes materiais é limitado ao preenchimento de defeitos ósseos nos quais o ambiente que o envolve é ainda osteoconductor. Nos casos onde a degeneração óssea é extensa, destas cerâmicas poucas o nenhuma vantagem regenerativa é oferecida já que degradam muito rápido como para dar um suporte para a formação óssea. ${ }^{2}$

Substitutos ósseos aloplásticos de fosfato de cálcio, como hidroxiapatita (HA) e fosfato tricálcico (TCP) têm sido estudados devido às semelhanças da sua composição com a fase inorgânica do osso. ${ }^{45}$ A própria fase mineral dos ossos é composta, principalmente, por hidroxiapatita, uma das cerâmicas mais utilizadas como enxerto ósseo e são basicamente osteocondutores. $^{2}$

A eficácia da hidroxiapatita é evidenciada em áreas de compressão e/ou apresentando inflamações, o que não acontece com o TCP e o octacalciofosfato isolados. Em geral, biomateriais a base de HA são considerados não reabsorvíeis ou de reabsorção lenta; enquanto os substitutos ósseos a base de TCP espera-se que possuam uma taxa de reabsorção mais rápida. Cientistas desenvolveram materiais mistos, também chamados de "compostos bifásicos", em que a HA teria a função de manter o arcabouço ósseo sem ser reabsorvida e o TCP ser substituído pelo novo tecido ósseo formado. ${ }^{2,23}$

A solubilidade do TCP em água é superior a da HA sob as mesmas condições. Assim se utilizam as duas combinadas em sistemas bifásicos para aumentar a solubilidade das cerâmicas em meio biológico aumentando sua reabsorção pelo corpo humano (regeneração / reabsorção). ${ }^{2}$

Estudos comparativos in vitro de diferentes proporções HA/TCP de fosfatos cálcios bifásico tem demostrado que a adesão celular de osteoblastos e osteoclastos / monócitos sobre a sua superfície é fortemente influenciada pela concentração de TCP, em que uma porcentagem aumentada de TCP reduz a adesão das células. ${ }^{23}$

Depois de uma exodontia, se exige um composto inorgânico de concentração maior de hidroxiapatita que outros a base de fosfato de cálcio. Tal necessida- de deve-se ao fato de existir um processo inflamatório após a cirurgia, mudando as condições de $\mathrm{pH}$ e metabólicas locais (presença de leucócitos polimorfonucleares), tal situação clinica desfavorece o tricalciofosfato, monocalciofosfato e outros pelas suas altas solubilidades em situações em que o $\mathrm{pH}$ varia. Na região anterior torna-se necessário a utilização de materiais mais rígidos e com pouca capacidade de reabsorção em conjunto com materiais de reabsorção rápida. ${ }^{23}$

Horowitz et al. ${ }^{46}$ observaram que depois de 6 meses os alvéolos preenchidos com $\beta$ - TCP mais membrana de colágeno, apresentavam uma perda óssea horizontal de $12 \%$. O rebordo foi preservado em espessura cerca do $91 \%$. E os cortes histológicos mostraram reabsorção do biomaterial e deposição do novo tecido ósseo.

Num estudo em cães compararam distintos tipos de recobrimento dos alvéolos com defeito na vestibular, enxertados com $\beta$-TCP (partículas de 0.5 - 1.5, porosidade de $75 \%$, tamanho de poros de $100 \mathrm{a} 400 \mu \mathrm{m}$ ). Quatro grupos foram avaliados: $\beta$ - TCP, $\beta$ - TCP recoberto com esponja de colágeno, $\beta$ - TCP recoberto com o retalho e sem tratamento. Depois de 12 semanas, os grupos enxertados com $\beta$ - TCP apresentaram em geral maior aumento da crista que o grupo controle. No estudo histológico, o grupo $\beta$ - TCP apresentou TCP rodeado de células inflamatórias, e não contínuas com a matriz óssea; o grupo $\beta$ - TCP + colágeno não apresentou vestígios do colágeno e o osso apresentou-se imaturo; no grupo $\beta$ - TCP + retalho manteve-se na crista, e a corticalização foi iniciada. O osso neoformado foi maior nos grupos enxertados ( $\beta$ - TCP: 8.4 $\mathrm{mm}^{2}, \beta$ - TCP + colágeno: $10.8 \mathrm{~mm}^{2} ; \beta$ - TCP + retalho $10.5 \mathrm{~mm}^{2}$ ) que no grupo controle $\left(4.8 \mathrm{~mm}^{2}\right) .{ }^{47}$

O estudo de Brkovic et al. ${ }^{48}$ mostrou que depois de 9 meses, os alvéolos que usaram $\beta$ - TCP e colágeno tipo I deixados expostos sem membrana nem retalho tiveram significativamente maior redução da dimensão horizontal do rebordo que os alvéolos preenchidos $\operatorname{com} \beta$ - TCP e colágeno tipo I mais membrana. Nos cortes histológicos, não houve diferença significativa entre o osso vital formado ( $42.4 \%$ sem membrana, $45.3 \%$ com membrana).

Wakimoto et al. ${ }^{49}$ estudaram o biomaterial Mastergraft (Medtronic), composto por $15 \%$ HA e $85 \% \beta$ 
- TCP, o biomaterial tem uma taxa de porosidade de $80 \%$ e uma média de tamanho de porosidade de 50 $\mu \mathrm{m}$. O corte histológico, depois de 12 semanas, mostrou uma neoformação óssea ao redor do Mastergraft e presença de tecido conjuntivo fibroso e osteócitos.

No estudo de Jensen et al. ${ }^{45}$ em mini-pigs, comparou-se quatro grupos de enxertos: autógenos, hidroxiapatita (HA), $\beta$ - tricalciofosfato (TCP) e um Bifásico HA/ TCP (60\% / 40\%). Observou-se que durante as quatro semanas todos os grupos tiveram diferença estatisticamente significativa na formação do novo osso (autógeno $>$ TCP $>\mathrm{HA} / \mathrm{TCP}>\mathrm{HÁ}$ ). Em oito semanas a quantidade de TCP pareceu influir na formação do osso, podendo ser devido a sua rápida absorção (autógeno e TCP > HÁ/TCP > HA). Nas 24 semanas, observou-se osso lamelar maduro com íntimo contato entre o enxerto e o osso, sem diferença significativa.

Crespi ${ }^{50}$ estudou alvéolos preservados com Sulfato de cálcio (CS), hidroxiapatita enriquecida com magnésio (MHA) e um grupo controle sem tratamento pós extração (GC). Três meses depois, o estudo histológico mostrou diferença significativa entre os grupos tendo uma média de osso vital no grupo CS de $40.0 \%$, MHA de $32.8 \%$ e C de $32.8 \%$. Observou-se uma redução do nível vertical do osso, o grupo MHA apresentou menor perda óssea $(0.48 \pm 0.21)$ que os grupos CS $(2.48 \pm 0.65)$ e C $(3.75 \pm 0.63)$.

Lindhe et al. ${ }^{51}$ num estudo em cães utilizou BPCAP, material bifásico, composto de um núcleo de TCP revestido de uma camada de espessura definida de hidroxiapatita, obtida da mistura de pós de cálcio e fosfato em proporções adequadas e sintetizados a altas temperaturas. BPCAP apresenta partículas altamente porosas $0.4-1 \mathrm{~mm}$, o tamanho do cristal é de $18 \times 18$ x $38 \mathrm{~nm}$ similar ao osso mineral natural e ambas fases apresentam uma cristalização menor a 95\%. A utilização de enxerto aloplastico bifásico não sofreu uma marcada reabsorção durante a modelagem/ remodelagem dos tecidos e permitiu a formação de grandes quantidades de osso no alvéolo. Assim o enxerto contrariaria a reabsorção da crista que naturalmente ocorre depois da extração dentária.

No estudo de Toloue ${ }^{52}$ onde comparou-se alvéolos enxertados com Sulfato de cálcio (CS) e com osso liofilizado (FDBA), não houve diferenças estatisticamente significativas entre as mudanças verticais e horizontais dos rebordos. Depois de três meses, os cortes histológicos mostraram que o grupo CS apresentava maior porcentagem de osso neoformado e osteóides que o grupo FDBA (31.74\% vs $16.66 \%$ ); também observou-se uma maior presença de enxerto remanescente no grupo FDBA que no grupo CS, sendo em alguns casos inexistente $(21.37 \%$ vs $2.54 \%)$.

Os vidros bioactivos ou biovidros (BG) são um tipo de cerâmica reabsorvível usada para reparos de tecidos duros do corpo. Apresentam uma composição fundamentada na presença de óxidos de cálcio, fósforo e silício. Várias combinações possíveis são comercializadas e estudadas atualmente. Acredita-se que o silício é importante na bioatividade. ${ }^{2,3}$

O primeiro biovidro comercial, usado até hoje, foi o Bioglass (BG). Estes biovidros de acordo com sua fabricação, podem apresentar-se como dispositivos densos em forma de grânulos ou em forma de solução gel. A regeneração óssea de defeitos tratados com grânulos de vidro é relatada como uma das mais satisfatórias dentre as cerâmicas implantáveis. ${ }^{2}$

Os BG são biomateriais baseados em compostos de silicato amorfos. Quando são implantados eles reagem para formar núcleos internos de gel de sílica com superfícies de fosfato de cálcio. Neste processo, o núcleo de gel de sílica degrada-se deixando grandes quantidades de fosfato de cálcio externo, e dentro dos grânulos escavados, células osteoprogenitoras se diferenciam e formam novo tecido ósseo. ${ }^{53}$

Santos et al.$^{53}$ num estudo em cães, mostraram em cortes histológicos que a cicatrização sem nenhum tipo de enxertia em 4 semanas formou osso lamelar e em 28 semanas apresentou osso compacto e formação de periósteo. Os alvéolos enxertados com HA artificial mostraram nas 4 semanas poucas células inflamatórias sem indução de resposta inflamatória, nas 8 semanas o biomaterial apresentou-se envolto num tecido fino calcificado e ao redor tecido conjuntivo, depois de 28 semanas observou-se o mesmo mas a maioria apresentava-se envolta em tecido ósseo. Não foi observada a completa reabsorção do biomaterial. Nos alvéolos enxertados com HA natural (osso bovino mineral) observou-se nas 4 semanas tecido conjuntivo fibroso 
com colágeno envolvendo as partículas do biomaterial parcialmente reabsorvido. Depois de 8 semanas, observou-se a presença de osteócitos envolvidos no biomaterial, e depois de 28 semanas observaram-se envoltos de tecido ósseo e alguns em tecido conectivo fibroso. Nos alvéolos enxertado com BG, observou-se células inflamatórias, nas 4 semanas seguintes o biomaterial apresentou algumas fissuras e infiltração celular, na 8 semana, observou-se o biomaterial com zonas de reabsorção e uma leve reação inflamatória. Nas 28 semanas, observou-se algumas amostras sem biomaterial e com osso compacto e outras amostras com o biomaterial envolto em uma camada delgada de tecido calcificado. O BG apresentou maior quantidade de partículas de biomaterial envoltas com tecido ósseo. Todos biomateriais interferiram no processo de reparação óssea.

\subsection{Combinação de biomateriais}

Considerando que os biomateriais existentes nenhum atinge todos os requisitos desejados, procurando-se enxertos compostos que em conjunto apresentem característica regenerativas mais complexas. $\mathrm{O}$ foco dos estudos clínicos na atualidade está na associação de diferentes arcabouços osteocondutores com fatores de crescimentos osteoindutores e/ ou células osteogênicas. ${ }^{2}$

Os enxertos compostos apresentam benefícios adicionais como: 1. Capacidade de osteocondução, osteoindução e osteogenicidade; 2. Modulação das características e propriedades do enxerto, de acordo com as necessidades clinica de cada paciente; 3 . Propriedades imunogênicas; 4 . Controles de qualidade e esterilidade; e 5. Disponibilidade. As principais desvantagem são o custo de produção, armazenamento e distribuição mais elevados destes produtos. ${ }^{2}$

Nas células tronco mesenquimais (CTMs) o efeito mais impressionante da atividade de proteína morfogenetica (BMPs) é a capacidade de induzir a diferenciação dessas células em osteoblastos maduros. Baixas concentrações de BMPs promovem sua diferenciação em adipócitos, enquanto altas concentrações destas proteínas promovem diferenciação em osteoblastos. Além disso, mostra-se que rhBMP-2 bloqueia a diferenciação de células mesenquimais em adipócitos. ${ }^{2,54}$
O plasma rico em plaquetas (PRP) promove a cicatrização do enxerto ósseo e dos tecidos moles com boa epitelização. PRP esta sendo utilizado para fornecer fatores de crescimento em concentração elevada para o osso receptor. ${ }^{55}$

$\mathrm{Na}$ atualidade, o PRP oferece vantagens como: 1 . Diminui a frequência do sangramento transoperatório e pós-operatório da área receptora. 2. Ajuda na estabilidade inicial do tecido enxertado na área enxertada como resultado de sua natureza coesiva e adesiva. 3. Pode promover rápida vascularização do tecido de cicatrização, fornecendo fatores de crescimento. 4 . Em combinação com biomateriais de substituição óssea, induz a regeneração de tecidos duros e moles. ${ }^{55}$

Suba et al ${ }^{56}$ num estudo em cães demostraram que os alvéolos enxertados com $\beta$-TCP + PRP apresentaram maior regeneração óssea em estágios iniciais que os enxertado só com $\beta$-TCP. Nas 12 semanas o grupo testado apresentou-se mais acelerado na regeneração óssea, observando material osteóides e matriz óssea penetrada no centro do biomaterial desintegrado. Depois de 24 semanas a atividade de neoformação óssea foi parecida em ambos grupos, a maioria de grânulos do biomaterial estão embebidos no novo osso lamelar formado.

No estudo de Kutkut et al..$^{55}$ foram avaliados alvéolos enxertados com sulfato de cálcio hemidratado de grau médio (MGCSH) + PRP cobertos com uma membrana de colágeno intencionalmente exposta versus os alvéolos enxertados com PRP livre com tampão de colágeno reabsorvível. Depois de 3 meses, o grupo MGCSH + PRP apresentou maior densidade na radiografia que o grupo controle, no estúdio histológico apresento maior quantidade de osso vital neoformado ( $66.5 \% \pm 10.4 \%$ ) que o grupo controle $(38.3 \%$ $\pm 9.3 \%$ ), os cortes histológicos do grupo testado apresentam-se com $100 \%$ de osso vital, bem mineralizado e organizado em trabéculas com diferença do grupo controle disposto em forma irregular. Observou-se aumento vertical na vestibular estatisticamente significativo do grupo testado de $0.6 \mathrm{~mm}$ versus a perda de $1.4 \mathrm{~mm}$ do grupo controle.

Nevins ${ }^{57}$ estudou alvéolos com defeitos ósseo na parede vestibular enxertados com MCBS e fator de crescimento recombinado derivado de plaquetas fator - BB (rhPDGF-BB, Gem21S, Osteohealth, que e 
PDGF em uma matriz de $\beta$-TCP) e sem membrana. Ao que parece a combinação deles, regenerou a parede vestibular óssea. Tanto em tempo de 4 e 6 meses, se observou a cascada de eventos de maturação óssea esperada, apresentando nova formação óssea e intimo contato com os remanescente de MCBS, separados por linhas de desmineralização.

MCAllister ${ }^{58}$ não encontrou diferença da nova formação óssea depois de três meses nos alvéolos enxertados com rhDPGF $+\beta$-TCP ou com rhPDGF$\mathrm{BB}+$ xenoenxerto de osso bovino desproteinizado inorgânico colágeno (bioOss Collagen), pôde-se observar na avaliação histológica que em ambos grupos uma maior formação óssea na apical das amostras que na área coronal, sendo coronalmente maior no grupo com xenoenxerto que no grupo com TCP.

\section{DISCUSSÃO}

A indicação do biomaterial em alvéolos pós extração esta relacionada à preservação do reborde do sítio de extração. ${ }^{59}$ Vários fatores devem ser avaliados ainda antes da extração: história clinica do paciente ${ }^{4}$, história dos dentes conhecendo a razão que levou o dente à extração, dentes com tratamento endodônti$\mathrm{Co}^{4,5}$, o estado periodontal, o biotipo periodontal ${ }^{5,60} \mathrm{e}$ finalmente, fatores estéticos. ${ }^{4}$

Depois da extração dentaria, uma perda óssea tanto horizontal como vertical é esperada, sendo mais severas nos primeiros 3 a 4 meses. ${ }^{8,10,11}$ Consequentemente o efeito se extenderá nos tecidos moles. A literatura mostra que a perda óssea horizontal é mais severa que a perda óssea vertical. Assim como, pelas características do alvéolo, esta reabsorção é maior na tábua óssea vestibular que na palatina/ lingual. ${ }^{8,911,61,62}$ Encontrou-se também, maiores perdas ósseas observadas em extrações múltiplas, e em região de molar quando comparado com região anterior, contudo a perda óssea nesta ultima é mais crítica pela tabua vestibular fina que apresenta e a estética exigida da região. ${ }^{8,13,63,64}$

Para a seleção do biomaterial que servirá como substituto ósseo é importante definir os objetivos à atingir com a enxertia, priorizá-los e fazer a escolha baseada no conhecimento das características de cada um e fatores ambientais como a disponibilidade e valor aquisitivo ao paciente. ${ }^{17}$

O enxerto de osso autógeno foi considerado como o padrão ouro durante muitos anos devido a suas propriedades de osteogêneses, osteocondutoras e osteoindutivas, já que contém células osseoprogenitoras, matriz extracelular colágena/ não colágena e diferentes fatores de crescimento e diferenciação. ${ }^{6}$ Porem, a morbilidade deste enxerto, en quanto a maior tempo da intervenção e processo inflamatórios em outra região, direciona à utilização de biomateriais, os quais oferecem também resultados favoráveis. ${ }^{59}$

Observa-se que os biomateriais alográficos são ostecondutores, mostrando atividade osteoblásticas nos cortes histológicos a partir das 6 semanas $^{17}$, ainda e apresentando resíduos do biomaterial que permanecem em estudo 19 semanas depois. ${ }^{29}$ Mostra-se uma reabsorção horizontal nos alvéolos enxertados tanto com FDBA e DFDBA,,$^{25,28,29}$ e no sentido vertical se mostra uma perda menor a $1 \mathrm{~mm}$ com FDBA e DFD$\mathrm{BA}^{29}$ ou um ganho mínimo em alguns casos FDBA. ${ }^{28}$

Estudos mostram nos cortes histológicos depois de 4 a 6 meses um íntimo contato entre o osso neoformado e as partículas do xenóenxerto, promovendo um osso organizado, ${ }^{30}$ não entanto a formação óssea comparada com os alvéolos não enxertados é menor. ${ }^{34}$ Observa-se também menor porcentagem de osso lamelar e medular que nos alvéolos sem tratamento. ${ }^{36}$ Estudos confirmam que esta cicatrização é mais rápida nas áreas apical e lateral do alvéolo que nas áreas marginal e central do mesmo. ${ }^{37}$ Isto explicaria o porquê da redução menor do rebordo alveolar. ${ }^{35}$

Vários a autores estudam as vantagens e desvantagens da hidroxiapatita (HA) e TCP. A HA tem uma solubilidade menor que o $\mathrm{TCP}^{2}$ e ela se mantém ainda em situações onde o $\mathrm{pH}$ variável como nos estados de inflamação o compressão depois da exodontia. ${ }^{2,23} \mathrm{~A}$ HA é considerada de reabsorção lenta enquanto que TCP tem uma reabsorção mais rápida sendo ideal para manter as dimensões do rebordo. ${ }^{2,23}$ Assim se estas são combinadas em compostos bifásicos com o fim de que HÁ mantenha o arcabouço ósseo e o TCP seja substituído pelo novo osso formado. 
Estudos em humanos ${ }^{55}$ e animais ${ }^{56}$ demostram que a associação de biomateriais de enxertia com outros fatores como o plasma rico em fibrina parece resultar em uma regeneração óssea mais intensa e em menor tempo.

\section{CONCLUSÕES}

Apesar de haver evidências cientificas para a presente revisão de literatura, estas são consideradas não conclusivas, em razão à heterogeneidade metodológica dos estudos. Contudo, a literatura é clara em mostrar os benefícios nos grupos preservados com biomateriais quando comparados com a cicatrização espontânea a respeito da perda óssea. Os biomateriais apresentam qualidades desejadas para o preenchimento do alvéolo pós-extração, as quais foram apresentadas no presente trabalho, porém precisam ser revisadas para cada caso clínico particular. No entanto, para o processo de tomada de decisões, é necessário analisar o custo e o desejo do paciente, pois a força da recomendação será determinada pelas necessidades individuais do paciente.

\section{REFERENCES}

1. Fardin AC, Jardim Ellen CG, Pereira FC, Guskuma MH, Aranega AM, Garcia Júnior IR. Enxerto ósseo em odontologia: revisão de literatura. Innov. Implant. J., Biomater. Esthet. 2010;5(3):48-52.

2. Granjeiro, J. M., Soares, G. D. A. de, Biomateriais em odontologia: princípios, métodos investigativos e aplicações, $1^{\text {a }}$ ed. São Paulo: VM Cultural Editora; 2011, p. 207.

3. Buser, D. 20 Years of guided bone regeneration in implant dentistry, 2da ed.Singapore: Quistessence; 2009, p. 261.

4. Caplanis N, Lozada JL, Kan JY. Extraction defect assessment, classification, and management. J Calif Dent Assoc. 2005;33(11):853-63.

5. Melnick, P. R.; Camargo, P. M. Alveolar bone preservation following tooth extraction in the esthetic zone. In: Implant and Regenerative Therapy in Dentistry: A Guide to Decision Making. $1^{\text {a }}$ ed. Iowa: Wiley- Blackwell. 2009. Cap. 10, p. 272-294.

6. Fugazzotto, P. A., Implant and Regenerative Therapy in Dentistry: A Guide to Decision Making, $1^{a}$ ed., Iowa: Wiley- Blackwell; 2009.
7. Pagni G, Pellegrini G, Giannobile WV, Rasperini G. Posextraction aveolar ridge preservation: biological basis and treatments. Int J Dent. 2012:1-13. doi: $10.1155 / 2012 / 151030$.

8. Pietrokovski J, Massler M. Alveolar ridge resorption following tooth extraction. J Prosthet Dent. 1967;17(1):21-7.

9. Araújo MG, Lindhe J. Dimensional ridge alterations following tooth extraction. An experimental study in the dog. J Clin Periodontol. 2005;32(2):212-8. doi: 10.1111/j.1600-051X.2005.00642.x.

10. Johnson K. A study of the dimensional changes occurring in the maxilla following tooth extraction. Aust Dent J. 1969;14(4):241-4. doi: 10.1111/j.1834-7819.1969. tb06001.x.

11. Schropp L, Wenzel A, Kostopoulos L, Karring T. Bone healing and soft tissue contour changes following single-tooth extraction: a clinical and radiographic 12-month prospective study. Int J Periodontics Restorative Dent. 2003;23(4):313-23.

12. Chen ST, Wilson TG Jr, Hämmerle CH. Immediate or early placement of implants following tooth extraction: review of biologic basis, clinical procedures, and outcomes. Int J Oral Maxillofac Implants. 2004;19 Suppl:12-25.

13. Nemcovsky CE, Serfaty V. Alveolar ridge preservation following extraction of maxillary anterior teeth. Report on 23 consecutive cases. J Periodontol. 1996;67(4):390-5. doi: 0.1902/jop.1996.67.4.390.

14. 14. Rasperini G, Canullo L, Dellavia C, Pellegrini G, Simion M. Socket grafting in the posterior maxilla reduces the need for sinus augmentation. Int J Periodontics Restorative Dent. 2010;30(3):265-73.

15. Nevins M, et al. A study of the fate of the buccal wall of extraction sockets of teeth with prominent roots. Int $\mathrm{J} \mathrm{Pe}$ riodontics Restorative Dent. 2006;26(1):19-29.

16. Block MS, Jackson WC. Techniques for grafting the extraction site in preparation for dental implant placement. Atlas Oral Maxillofac Surg Clin North Am. 2006;14(1):125. doi: 10.1016/j.cxom.2005.11.006.

17. Santos FA, Pochapski MT, Martins MC, Zenóbio EG, Spolidoro LC, Marcantonio E Jr. Comparison of biomaterial implants in the dental socket: histological analysis in dogs. Clin Implant Dent Relat Res. 2010;12(1):18-25. doi: $10.1111 /$ j.1708-8208.2008.00126.x.

18. Darby I, Chen S, De Poi R. Ridge preservation: what is it and when should it be considered. Aust Dent J. 2008;53(1):11-21. doi: 10.1111/j.1834-7819.2007.00008.x. 
19. Hupp, J. R.; Ellis, III E.; Tucker, M. R.; Cirurgia Oral e Maxilofacial Contemporânea. Elsevier, $5^{\mathrm{a}}$ ed. Rio de Janeiro: Elsevier; 2009. P. 53.

20. Farina, R.; Trombelli L. Wound healing of extraction sockets. Endod topics. 2011;25 (1):16-43. doi: 10.1111/ etp. 12016 .

21. Cardaropoli G, Araújo M, Lindhe J. Dynamics of bone tissue formation in tooth extraction sites. An experimental study in dogs. J Clin Periodontol. 2003;30(9):809-18. doi: 10.1034/j.1600-051X.2003.00366.x.

22. Carvalho, P. S. P. de. et al. Biomaterias aplicados a implantodontia. In: Fundamentos em implantodontia: uma visão contemporânea, 1 ed., São Paulo: Quintessence; 2011, cap. 8, p. $111-123$.

23. De Carvalho, P. S. P. Subtitutos Osseos- Quando Utilizá-los? In: Osseointegração 20 anos: Visão contemporânea da Implantodontia. 1ra ed. São Paulo: Quintessence; 2009. cap 7. P. 197-122.

24. Becker W, Becker BE, Caffesse R. A comparison of demineralized freeze-dried bone and autologous bone to induce bone formation in human extraction sockets. J Periodontol. 1994 Dec;65(12):1128-33. doi: 10.1902/ jop.1994.65.12.1128.

25. Brownfield LA, Weltman RL. Ridge preservation with or without an osteoinductive allograft: a clinical, radiographic, micro-computed tomography, and histologic study evaluating dimensional changes and new bone formation of the alveolar ridge. J Periodontol. 2012;83(5):581-9. doi: 10.1902/jop.2011.110365.

26. Scheyer ET, Schupbach P, McGuire MK. A histologic and clinical evaluation of ridge preservation following grafting with demineralized bone matrix, cancellous bone chips, and resorbable extracellular matrix membrane. Int J Periodontics Restorative Dent. 2012;32(5):543-52.

27. Beck TM, Mealey BL. Histologic analysis of healing after tooth extraction with ridge preservation using mineralized human bone allograft. J Periodontol. 2010;81(12):1765-72. doi: 10.1902/jop.2010.100286.

28. Lasella JM et al. Ridge preservation with freeze-dried bone allograft and a collagen membranecompared to extraction alone for implant site development: a clinical and histologic study in humans. J Periodontol. 2003;74(7):990-9. doi: 10.1902/jop.2003.74.7.990.

29. Wood RA, Mealey BL. Histologic comparison of healing after tooth extractionwith ridge preservation using mineralized versus demineralized freeze-dried bone allograft. J Periodontol. 2012;83(3):329-36. doi: 10.1902/ jop.2011.110270.

30. Lee DW, Pi SH, Lee SK, Kim EC. Comparative histomorphometric analysis of extraction sockets healing implanted with bovine xenografts, irradiated cancellous allografts, and solvent-dehydrated allografts in humans. Int J Oral Maxillofac Implants. 2009;24(4):609-15.

31. Barone A, Aldini NN, Fini M, Giardino R, Calvo Guirado JL, Covani U. Xenograft versus extraction alone for ridge preservation after tooth removal: a clinical and histomorphometric study. J Periodontol. 2008;79(8):1370-7. doi: 10.1902/jop.2008.070628.

32. Ten Heggeler JM, Slot DE, Van der Weijden GA. Effect of socket preservationtherapies following tooth extraction in non-molar regions in humans: a systematic review. Clin Oral Implants Res. 2011;22(8):779-88. doi: 10.1111/j.1600-0501.2010.02064.x.

33. Kim YK, Yun PY, Lee HJ, Ahn JY, Kim SG. Ridge preservation of the molar extraction socket using collagen sponge and xenogeneic bone grafts. Implant Dent. 2011;20(4):267-72. doi: 10.1097/ID.0b013e3182166afc.

34. Heberer S, Al-Chawaf B, Jablonski C, Nelson JJ, Lage H, Nelson K. Healing of ungrafted and grafted extraction sockets after 12 weeks: a prospective clinical study. Int J Oral Maxillofac Implants. 2011;26(2):385-92.

35. Cardaropoli D, Tamagnone L, Roffredo A, Gaveglio L, Cardaropoli G. Socket preservation using bovine bone mineral and collagen membrane: a randomized controlled clinical trial with histologic analysis. Int J Periodontics Restorative Dent. 2012;32(4):421-30.

36. Lindhe J, Cecchinato D, Donati M, Tomasi C, Liljenberg B. Ridge preservation with the use of deproteinized bovine bone mineral. Clin Oral Implants Res. 2014;25(7):78690. doi: 10.1111/clr.12170.

37. Araújo MG, Liljenberg B, Lindhe J. Dynamics of Bio-Oss Collagen incorporation in fresh extraction wounds: an experimental study in the dog. Clin Oral Implants Res. 2010;21(1):55-64. doi: 10.1111/j.1600-0501.2009.01854.x.

38. Perelman-Karmon M, Kozlovsky A, Liloy R, Artzi Z. Socket site preservation using bovine bone mineral with and without a bioresorbable collagen membrane. Int J Periodontics Restorative Dent. 2012;32(4):459-65.

39. Crespi R, Capparè P, Gherlone E. Comparison of magnesium-enrichedhydroxyapatite and porcine bone in human extraction socket healing: a histologic and histomorphometric evaluation. Int J Oral Maxillofac Implants. 2011;26(5):1057-62. 
40. Mardas N, Chadha V, Donos N. Alveolar ridge preservation with guided boné regeneration and a synthetic bone substitute or a bovine-derived xenograft: a randomized, controlled clinical trial. Clin Oral Implants Res. 2010;21(7):68898. doi: 10.1111/j.1600-0501.2010.01918.x.

41. Gholami GA, Najafi B, Mashhadiabbas F, Goetz W, Najafi S. Clinical, histologic and histomorphometric evaluation of socket preservation using a synthetic nanocrystalline hydroxyapatite in comparison with a bovine xenograft: a randomized clinical trial. Clin Oral Implants Res. 2012;23(10):1198-204. doi: 10.1111/j.1600-0501.2011.02288.x.

42. Berube P, Yang Y, Carnes DL, Stover RE, Boland EJ, Ong JL. The effect of sputtered calcium phosphate coatings of different crystallinity on osteoblast differentiation. J Periodontol. 2005;76(10):1697-709. doi: 10.1902/ jop.2005.76.10.1697.

43. Rohanizadeh R, Padrines M, Bouler JM, Couchourel D, Fortun Y, Daculsi G. Apatite precipitation after incubation of biphasic calcium-phosphate ceramic invarious solutions: influence of seed species and proteins. J Biomed Mater Res. 1998;42(4):530-9. Doi: 10.1002/(SICI)1097-46 36(19981215)42:4<530::AID-JBM8>3.0.CO;2-6.

44. Ruga E, Gallesio C, Chiusa L, Boffano P. Clinical and histologic outcomes of calcium sulfate in the treatment of postextraction sockets. J Craniofac Surg. 2011;22(2):4948. doi: 10.1097/SCS.0b013e318208bb21.

45. Jensen SS, Yeo A, Dard M, Hunziker E, Schenk R, Buser D. Evaluation of a novel biphasic calcium phosphate in standardized bone defects: a histologic and histomorphometric study in the mandibles of minipigs. Clin Oral Implants Res. 2007;18(6):752-60. doi: 10.1111/j.1600-0501.2007.01417.x.

46. Horowitz RA, Mazor Z, Miller RJ, Krauser J, Prasad HS, Rohrer MD. Clinical evaluation alveolar ridge preservation with a beta-tricalcium phosphate socket graft. Compend Contin Educ Dent. 2009;30(9):588-90.

47. Inomata K, Marukawa E, Takahashi Y, Omura K. The effect of covering materials with an open wound in alveolar ridge augmentation using beta-tricalcium phosphate: an experimental study in the dog. Int J Oral Maxillofac Implants. 2012;27(6):1413-21.

48. Brkovic BM, Prasad HS, Rohrer MD, Konandreas G, Agrogiannis G, Antunovic D, Sándor GK. Beta-tricalcium phosphate/type I collagen cones with or without a barrier membrane in human extraction socket healing: clinical, histologic, histomorphometric, and immunohistochemical evaluation. Clin Oral Investig. 2012;16(2):581-90. doi: 10.1007/s00784-011-0531-1.
49. Wakimoto M, Ueno T, Hirata A, Iida S, Aghaloo T, Moy PK. Histologic evaluation of human alveolar sockets treated with an artificial bone substitute material. J Craniofac Surg. 2011;22(2):490-3. doi: 10.1097/ SCS.0b013e318208bacf.

50. Crespi R, Capparè P, Gherlone E. Magnesium-enriched hydroxyapatite compared to calcium sulfate in the healing of human extraction sockets: radiographic and histomorphometric evaluation at 3 months. J Periodontol. 2009;80(2):210-8. doi: 10.1902/jop.2009.080400.

51. Lindhe J, Araújo MG, Bufler M, Liljenberg B. Biphasic alloplastic graft used to preserve the dimension of the edentulous ridge: an experimental study in the dog. Clin Oral Implants Res. 2013;24(10):1158-63. doi: 10.1111/j.1600-0501.2012.02527.x.

52. Toloue SM, Chesnoiu-Matei I, Blanchard SB. A clinical and histomorphometric study of calcium sulfate compared with freeze-dried bone allograft for alveolar ridge preservation. J Periodontol. 2012;83(7):847-55. doi: 10.1902/ jop.2011.110470.

53. Santos FA, Pochapski MT, Martins MC, Zenóbio EG, Spolidoro LC, Marcantonio E. Jr. Comparison of biomaterial implants in the dental socket: histological analysis in dogs. Clin Implant Dent Relat Res. 2010;12(1):18-25. doi: 10.1111/j.1708-8208.2008.00126.x.

54. Tunes, R. U. da; Dourado, M.; Bitterncourt, S. Terapêutica periodontal regenerativa: técnicas cirúrgicas, biomateriais e fatores de crescimento transformando o periodontista em engenheiro tecidual. In: Avanços em periodontia e implantodontia: paradigmas e desafios, 1 ed., Nova Odessa: Napoleão; 2011. cap, p. 564-576.

55. Kutkut A, Andreana S, Kim HL, Monaco E Jr. Extraction socket preservationgraft before implant placement with calcium sulfate hemihydrate and platelet-rich plasma: a clinical and histomorphometric study in humans. J Periodontol. 2012;83(4):401-9. doi: 10.1902/jop.2011.110237.

56. Suba Z, Takács D, Gyulai-Gaál S, Kovács K. Facilitation of beta-tricalcium phosphate-induced alveolar bone regeneration by platelet-rich plasma in beagle dogs: a histologic and histomorphometric study. Int J Oral Maxillofac Implants. 2004;19(6):832-8.

57. Nevins ML, Camelo M, Schupbach P, Kim DM, Camelo JM, Nevins M. Human histologic evaluation of mineralized collagen bone substitute and recombinante platelet-derived growth factor-BB to create bone for implant placement in extraction socket defects at 4 and 6 months: a case series. Int J Periodontics Restorative Dent. 2009;29(2):129-39. 
58. McAllister BS, Haghighat K, Prasad HS, Rohrer MD. Histologic evaluation ofrecombinant human platelet-derived growth factor-BB after use in extractionsocket defects: a case series. Int J Periodontics Restorative Dent. 2010;30(4):365-73.

59. Hammerle $\mathrm{CH}$, Chen ST, Wilson TG. Consensus statements and recommended clinical procedures regarding the placement of implants in extraction socket. Int J Oral Maxillofa Implants. 2004;19 (Supl):26-8.

60. Sclar AG. Strategies for management of single-tooth extraction sites inaesthetic implant therapy. J Oral Maxillofac Surg. 2004 Sep;62(9 Suppl 2):90-105. Erratum in: J Oral Maxillofac Surg. 2004;62(9 Supl 2):90-105.

61. Covani U, Bortolaia C, Barone A, Sbordone L. Bucco-lingual crestal boné changes after immediate and delayed implant placement. J Periodontol. 2004;75(12):1605-12. doi: 10.1902/jop.2004.75.12.1605.
62. Avila-Ortiz G, Elangovan S, Kramer KW, Blanchette D, Dawson DV. Effect of alveolar ridge preservation after tooth extraction: a systematic review and meta-analysis. J Dent Res. 2014;93(10):950-8. doi: $10.1177 / 0022034514541127$.

63. Chappuis V, Araújo MG, Buser D. Clinical relevance of dimensional bone and soft tissue alterations post-extraction in esthetic sites. Periodontol 2000. 2017;73(1):73-83. doi: $10.1111 /$ prd.12167.

64. Januário AL, Duarte WR, Barriviera M, Mesti JC, Araújo MG, Lindhe J. Dimension of the facial bone wall in the anterior maxilla: a cone-beam computed tomography study. Clin Oral Implants Res. 2011;22(10):1168-71. doi: 10.1111/j.1600-0501.2010.02086.x. 